\title{
WALDDEGRADATION UND WALDSANIERUNG IM RAUM VON GARMISCH-PARTENKIRCHEN
}

\author{
Mit 7 Abbildungen, 5 Tabellen und 2 Kartenbeilagen (I + II)
}

\author{
FRIEDRICH REINER EHRIG
}

Summary: Forest degradation and restoration of forest lands in the Garmisch-Partenkirchen area.

Intensive grazing of forest lands until 1918, which has since decreased, combined with an increasingly excessive wildlife density resulted in extensive degradation of subalpine mountainous forests as well as in considerable disturbance of lower mixed-stand forests in the upland mountainous landscape of southern Bavaria in the area of Garmisch-Partenkirchen. The substantial decline in the protective function of forest lands for valley areas was expressed by increasing soil erosion and uncontrolled mountain stream activity. Until now the efforts to control mountain torrents along with local reforestation, in order to stabilize this landscape, have been limited to objectoriented protection measures. Further degradation of forests due to recently increasing use of these areas for sheep grazing, while maintaining high wildlife densities can only be prevented by consistent application of so called "integral restoration" of forest lands on a broad regional basis.

\section{Problemstellung}

Im gesamten Alpengebiet befindet sich die traditionelle Wirtschaftsform der Bergbauern in einem raschen Wandel, hauptsächlich verursacht durch den sich sprunghaft entwickelnden Fremden-verkehr. Damit erfährt auch der Wald dieser alten Kulturlandschaft einen
Funktionswandel. War bislang die Nutzungsform eine deutliche Vorrangfunktion des Waldes, so wird heute zunehmend die Schutzfunktion gegenüber den Talräumen gefordert. Da aber bekanntlich nur ein naturnaher Wald bei pfleglicher Bewirtschaftung diese Schutzfunktion erfüllen kann, andererseits bislang fast reine Nutzwälder vorliegen, muß erwartet werden, daß die heutigen Waldbestände der oberbayerischen Gebirgslandschaft nicht den Ansprüchen unserer Gesellschaft entsprechen.

Am Beispiel des alpinen Landschaftsraumes GarmischPartenkirchen, ehemals Bergbauerngebiet und heute internationales Fremdenverkehrszentrum, wird die gegenwärtige Situation der Wälder auf ihre Schutztauglichkeit untersucht. Wichtig erscheint hierbei die Erfassung der Hauptnutzung in Gestalt des Weideviehs und der Jagdnutzung. Im Anschluß an die Analyse des Waldzustandes wird auf die Notwendigkeit und Wirksamkeit der Sanierungsmaßnahmen in diesem Raum einzugehen sein.

\section{Bedeutungswechsel der Waldweide und Problematik des Wildbestandes}

Wie in allen Bergbauerngebieten der Alpen wurde auch im Raum Garmisch-Partenkirchen die Waldweide lange Zeit intensiv ausgeübt. Landläufig gilt die Waldweide als ein Gewohnheitsrecht, tatsächlich aber han- 


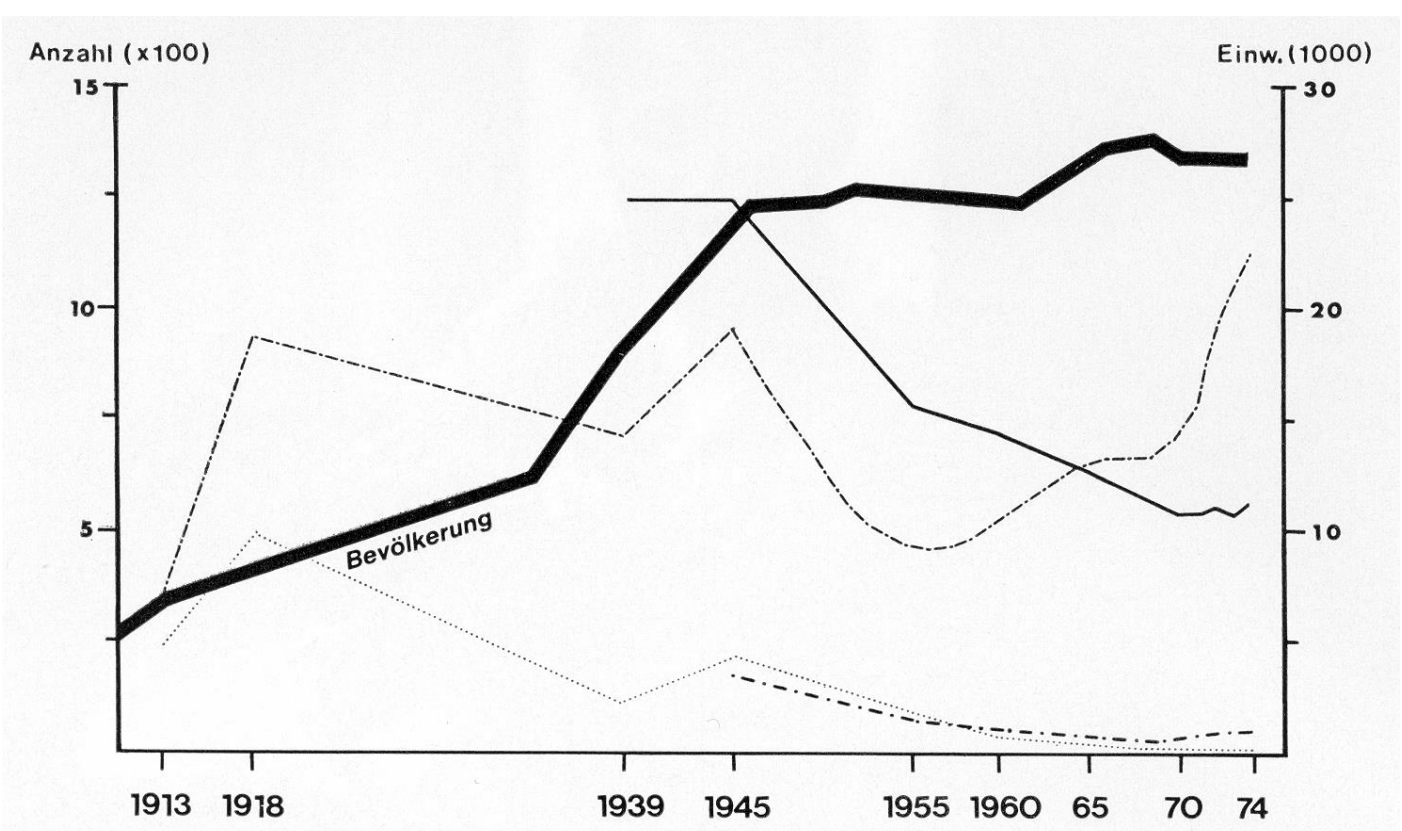

Abb. 1: Veränderung der Bevölkerung und des Viehbestandes von Garmisch-Partenkirchen

Changes in human population and livestock numbers in the Garmisch-Partenkirchen area

delt es sich um ein verbrieftes Weiderecht aus dem 15. Jahrhundert. In der 2. Waldordnung von 1408 sprach der Bischof von Freising den Einwohnern von Garmisch den Kramerstock und das Alpspitz-Kreuzeckgebiet als Waldweide $\mathrm{zu}$, den Bewohnern von Partenkirchen das Reintal, den Wettersteinwald und das Wankgebiet.

Die Forstrechte erschweren nicht nur die pflegliche Waldbewirtschaftung, sondern wirken in der Regel walddegradierend. Mit der Wandlung der Agrarverfassung der Bergbauerngemeinden verloren jedoch die Forstrechtsgrunddienst-barkeiten, vor allem die Waldweide und die Streurechte, zunehmend an Bedeutung.

Parallel zu dem starken Rückgang des Viehbestandes im Raum Garmisch-Partenkirchen, mit Ausnahme des Schafbestandes (Abb. 1), verlief die Ablösung der einzelnen Forstrechte. Von den 669 Waldweiderechten der ehemaligen Forstämter Partenkirchen und Garmisch wurden von 1848 bis 1976 insgesamt 311 (46\%) abgelöst. Die Streu-rechte gingen in diesem Zeitraum sogar um $96 \%$ zurück.

Die Belastung der Wälder im Untersuchungsgebiet durch die Waldweide zeigt die Karte (Beil. I) „Belastung der Landschaft und Teilsanierung" in welcher die Waldweiderechtsflächen verzeichnet sind. Insgesamt sind es 10 bewirtschaftete und erhaltungswürdige Berechtigungsalmen mit einer Gesamtfläche von 7.721

Der Viehauftrieb betrug 1975936 Rinder und 1.231 Schafe. Gegenüber 1972 verringerte sich der Rinderbestand um 5\%, während sich der Schafbestand um 59\% erhöhte. Dieser zunehmende Schafauftrieb verdeutlicht den Wandel in der Entwicklung der Almwirtschaft. Wie RUPPERT et. al., 1971 darstellen, vollzog sich im Laufe der letzten 120 Jahre nicht nur ein mehrfacher Wechsel in der Struktur der Almwirtschaft von der Galtvieh- über die Sennalm wieder zur Galtviehalm, sondern es verstärkte sich in jüngster Zeit außerdem der Rückgang der Almen. So war 1918 ein Höchststand des Viehbestandes, der sich seitdem ständig verringerte, lediglich von einem kleineren Maximum von 1945 unterbrochen. Diese bis 1965 rückläufige Tendenz der Almwirtschaft und damit auch der Waldweide erfuhr durch die zunehmende Extensivierung, insbes. durch die Schafhaltung einen deutlichen Wandel. 1965 dürfte die Waldweide nur mehr $1 / 3$ jener von 1918 ausgemacht haben; seitdem stieg sie jedoch auf knapp die Hälfte der Intensität von 1918 an. Welche enorme Auswirkung auf den Wald eine derartige Waldweide hatte, wird weiter unten diskutiert.

Von forstlicher Seite ist die erneute Zunahme der Waldweide unerwünscht, leider blieben einschränkende Maßnahmen ohne Erfolg, wie die Senkung der staatlichen Auftriebsprämie, oder werden umgangen, wie die maximalen Auftriebszahlen. So wurden beispielsweise 1974 im Reintal anstatt der zugelassenen 600 Schafe 800900 Tiere gesömmert.

War demnach die Waldweide seit der Jahrhundertwende insgesamt rückläufig, mit Ausnahme der jüngsten Entwicklung, und verlief somit zugunsten des Waldes, so wird sie jedoch von der gegenläufigen Entwicklung des Schalenwildbestandes überlagert. 
Leider läßt sich der Wildbestand früherer Jahrhunderte eben so wenig genau angeben wie die heutige Wilddichte. Stets handelt es sich hierbei um geschätzte Angaben bzw. Näherungswerte, welche mit z. T. beträchtlichen Unsicherheitsfaktoren belastet sind. Selbst die Abschußziffern, die man heute neben den Rotwildzählungen in den Wintergarten zur ungefähren Bestimmung des Wildbestandes verwendet, waren früher meist zu niedrig angegeben. Dennoch kann man aus den Abschußziffern die ungefähre Entwicklung des Wildbestandes ermitteln.

Bis in die zweite Hälfte des 16. Jh. dürfte der Rotwildbestand in den Bergwäldern Bayerns 10 Stück/1.000 ha betragen haben (v. KOBELL, 1858). Diese Wilddichte wird heute allgemein, wenn auch nicht zutreffend, wie später noch gezeigt wird, als natürlich , angesehen (J. FRÖHLICH, 1955; G. MEISTER, 1969).

Nach der Revolution von 1848 wurde aus der bislang Bischöflich-Freising'schen Jagd die Königliche Hofjagd Partenkirchen. Mit ihr setzte die Wildhege und besonders die Winterfütterung und die Schonung der Geisen und Kitze ein. Mit der Winterfütterung wollte man das Rotwild auch im Winter im Revier halten; früher war es im Herbst in die Auwälder des Voralpengebietes hinabgestiegen. Durch die Hege vergrößerte sich der Wildbestand erheblich, wie es die Jagdstrecken für die Verwaltungsjagden in Bayern angeben: 1908: 9.005 Stck. Schalenwild, 1936: 18.436 und 1961: 26.724. Betrug der Abschuß 1908 noch 6 bis 7 Stck. Rotwild/1.000 ha, so stieg er auf 13 Stck. 1961 bzw. 18 Stck. 1965. Der Wildbestand im Raum Garmisch-Partenkirchen zeigte hierbei die gleiche Entwicklung wie die des gesamten bayerischen Alpenraumes: der Rotwildabschuß erhöhte sich in 100 Jahren um das 8fache, von 1849 bis 1965 sogar um das 28 fache.

Von dem Abschuß kann man annähernd auf den Gesamtwildbestand schließen, wenn man davon ausgeht, daß der Abschuß i. d. Regel ca. 1/4 des Zuwachses ist (G. MEISTER, 1969). Nachdem 1858 der Rotwildabschuß im Raum Garmisch-Partenkirchen 1 Stck./1.000 ha betrug, dürfte der Rotwildbestand annähernd 10-12 Stck./1.000 ha betragen haben. Dieser Rotwildbestand wird von dem „Dermaligen Wildbestand im Bayerischen Hoch-gebirge; Forstverwaltung Bayerns, 1861“ bestätigt. Nach dem genannten Werk standen im Hochgebirge ferner je 1.000 Tagewerk 6 Rehe (17,6 Stck./1.000 ha) und 7 Gemsen (20,5/1.000 ha).

Im Forstamtsbereich Garmisch-Partenkirchen soll die tatsächliche Wilddichte folgende sein: 40 Stck. Rotwild/1.000 ha Rotwildfläche, 30 Stck. Rehwild/1.000 ha Rehwildfläche und 60 Stck. Gams-wild/1.000 ha Gamswildfläche. Wenn man beachtet, daß sich die Äsungsflächen des Rot- und Rehwildes und der Gemsen stark überschneiden und die drei Schalenwildarten zeitund gebietsweise als Äsungskonkurrenten auftreten, muß die wirkliche Wilddichte im Untersuchungsgebiet wesentlich über der von G. MEISTER, 1969 mit 55 Stck. angegebenen liegen. Die sehr präzisen Erhebungen von
W. DANZ, 1970 ergaben für den LK GarmischPartenkirchen tatsächlich höhere Werte (Tabelle 1).

Tabelle 1

\begin{tabular}{lcrrr}
\hline & $\begin{array}{l}\text { Rot- } \\
\text { wild }\end{array}$ & $\begin{array}{l}\text { Reh- } \\
\text { wild }\end{array}$ & $\begin{array}{l}\text { Gams- } \\
\text { wild }\end{array}$ & \multicolumn{2}{l}{$\begin{array}{l}\text { Ge- } \\
\text { samt }\end{array}$} \\
\hline Gesamtbestand (Stck.) & 3228 & 1591 & 3056 & 7875 \\
\hline Stck./1000ha & & & & \\
- Revierfläche & 39 & 19 & 37 & 95 \\
- Äsungsfläche & 55 & 27 & 52 & 135 \\
- Holzbodenfläche & 79 & 39 & 75 & 193 \\
\hline
\end{tabular}

Die nach den Lebensansprüchen der Tiere aufgeschlüsselte Waldfläche und somit ermittelte wirklich Wilddichte machen deutlich, mit welchen Schwächen generelle Angaben über die Wilddichte behaftet sind.

Für die Behandlung der Folgen der Veränderung des Wildbestandes auf den Wald kann man jedoch vorsichtig annehmen, daß sich der Wildbestand im Raum GarmischPartenkirchen seit 1860 mindestens verzehnfacht hat (dazu auch J. KARL, 1968).

\section{Die Schadwirkung von Waldweide und Wildäsung am Wald}

Das Beispiel des Mittelmeerraumes mit seinen devastierten Wäldern zeigt deutlich, welche verheerende Wirkung die Waldweide haben kann. Schafe und Ziegen sind hierbei, vor allem wegen ihrer Neigung alle Pflanzen intensiv zu verbeißen, bei den Forstleuten besonders gefürchtet. Hierbei ist allerdings zu beachten, daß sich der Viehverbiß je nach Tierart und außerdem je nach Vegetationsstufe recht unterschiedlich auswirkt. Ferner spielt die Exposition im Gebirge eine große Rolle: auf den frühzeitig aperen Südhängen ist die Weidedauer länger und die negative Auswirkung der Waldweide auf die Vegetation entsprechend größer als auf den Schatthängen. Generell gesehen bewirkt die Waldweide durch Almvieh nicht nur eine Abnahme der Stammzahl und des Holzvorrates, sondern vor allem auch eine wesentlich e Auflockerung des Waldes.

Waldweide und Almrodung zum Zweck der Vergrößerung der Lichtweiden sind für die Absenkung der Waldgrenze im Laufe der letzten Jahrhunderte verantwortlich. Beispielsweise liegt die Waldgrenze an der SE-Flanke des Kramers bei $1.400 \mathrm{~m}$ am tiefsten, im Mittel liegt sie sonst im Untersuchungsgebiet um ca. 250 $m$ höher (dazu K. HERMES, 1955). Die historische Waldgrenzdepression betrug im Raum GarmischPartenkirchen im Mittel $150 \mathrm{~m}$ (siehe auch Karte: Belastung der Landschaft und Teilsanierung um Garmisch-Partenkirchen). 
Die seit 1820 wesentlich verbesserte forstliche Bewirtschaftung der Wälder im Gebirge hatte günstigere Voraussetzungen der Wiederbegründung naturnaher Mischwälder geschaffen. Tatsächlich aber finden wir hier fast keinen naturnahen Mischwald mehr. Als Hauptursache dieser negativen Waldveränderung nimmt G. MEISTER, 1969 neben der Waldweide vor allem den Verbiß durch den stark angestiegenen Wildbestand an. Aus den Forsteinrichtungswerken des Forstamtes Partenkirchen können wir bereits 1929 entnehmen, daß damals der Wildverbiß das Wirtschaftsziel eines naturnahen Mischwaldes gefährdete.

Wie kommt es eigentlich $\mathrm{zu}$ dieser negativen Auswirkung der Wildäsung? Die Nahrung des Wildes setzt sich aus weicher (Gras, Kräuter) und zäher Äsung (Zweige, Sämlinge, Baumrinden) zusammen. Die zähe Äsung ist für das Rotwild während des Sommers wichtige Zusatznahrung (30\%), Reh- und Gamswild benötigen selbst im Sommer ca. 60\% zähe Äsung (H. MAYER \&. A. STEINHÄUSER, 1967). Im Winter ist der Bedarf an zäher Äsung entsprechend höher. Trotz der Winterfütterungen und den Rotwildgatterungen dürfte der Verbissschaden erheblich sein, da sich Reh- und Gamswild nicht gattern lassen.

Die Äsungsschäden wurden in den Forstämtern Reit im Winkl und Reichenhall-Süd auf insgesamt 7.040 qm studiert (G. MEISTER, 1969). Es ergab sich, daß in den gezäunten Flächen sehr schnell eine naturnahe Vegetation aufkam, während außerhalb derselben die Pflanzen stark verbissen waren. Die Vegetationsaufnahmen der Probeflächen bestätigen den selektiven Verbiß des Wildes. Am stärksten verbissen werden Holunder, Bergahorn, Mehlbeere, Aspe, Esche, Erle, Ulme und Tanne, gefolgt von Vogelbeere, Heide und Heidelbeere. Sobald die bevorzugten Pflanzen dezimiert sind, greift die Wildäsung auch auf Fichten und Buchen zurück. Die Latschen werden, offenbar wegen ihres hohen Harzgehaltes, weniger verbissen. Wie bereits erwähnt, spielt bei der Äsung die Schneedecke eine wichtige Schutzrolle hinsichtlich der Vegetation und zwar sowohl nach Schneehöhe als auch ihrer Dauer: auf den Sonnenseiten kann praktisch keine Laubholzart mehr aufkommen.

Außer den Verbißschäden sind noch die Schälschäden, d. h. das Abschälen der Rinde zu nennen. Nach W. LAATSCH u. W. GROTTENTHALER, 1973 werden sie umso katastrophaler, je mehr sich mit dem Rückgang des Laubholzanteils unserer Gebirgswälder das Äsungsangebot verringert. Schälschäden sind forstwirtschaftlich besonders gravierend, da die geschälten Bäume wuchsgestört sind und $\mathrm{zu}$ vorzeitigem Abtrieb der Bestände zwingen (R. A. JugOVIZ, 1908; G. FISCHBACHER, 1956; W. MANTEL, 1925). Bereits 1949 ermittelte R. MAGIN die Zuwachsminderung durch die Wildäsung (Abb. 2). Im Durchschnitt ist sie bei beweideten Flächen um $41 \%$ geringer als auf standörtlich vergleichbaren und nicht beweideten Standorten. Welche

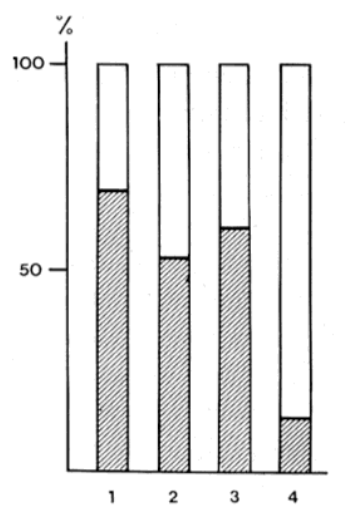

Abb.2: Die Wirkung der Waldweide auf Zuwachs und Ertrag des Waldes (R. MAGIN, 1949)

Alle Größen der unbeweideten Fläche sind gleich 100 gesetzt und die Größen der beweideten Flächen in Prozent ausgedrückt; Mittelwerte aller Untersuchungsflächen. 1 Mittelhöhe, 2 Stammzahl, 3 Ges. Kreisfläche, 4 Natürliche Verjüngung.

Impact of grazing forest lands on growth rates and production of timber

All units of ungrazed areas equal 100, the size of grazed lands is expressed in relation to 100; median values of all study areas. 1 median altitude, 2 numbcr I tree trunks, 3 total area of Kreis, 4 natural rejuvenation

wertmäßige Größenordnung die Schäden durch die Wildäsung in dem sehr langsam wüchsigen Berg- und Gebirgswald ausmachen, konnte bislang noch nicht ermittelt werden. Außer Zweifel steht jedoch, daß sowohl Waldweide als auch Wildäsung, abgesehen von weiteren Waldschädigungen wie z. B. Trittschäden durch größere Populationen oder Überdüngung, nicht nur eine beachtliche forstwirtschaftliche Leistungsminderung des Waldes, sondern vor allem landschaftsökologisch unvertretbar hohe Schäden verursachen. Das Ausmaß der Walddegradation und ihre Folgen durch Waldweide und Wildverbiß soll im Folgenden für den Raum GarmischPartenkirchen dargelegt werden.

\section{Ausmaß und Folgen der Walddegradation}

Waldweide und vor allem überhöhte Wilddichte müssen sich negativ auf den Waldaufbau auswirken (Abb. 3). Der Anteil der Mischbestände in den Schutz- und Wirtschaftswäldern des Staatswaldes im bayerischen Hochgebirge ging von 52\% bzw. 59\% um 1821 auf gegenwärtig $2 \%$ bzw. $4 \%$ zurück und dies trotz der forstlichen Zielsetzung Fichten- Buchen- TannenMischwälder zu begründen. Die Abnahme der Mischbestände vollzog sich in den fast gänzlich in der Montanstufe gelegenen Wirtschaftswäldern von 1820 bis 1900 relativ gleichmäßig auf $15 \%$, um sich seitdem langsamer auf $4 \%$ zu verringern. Die Schutzwälder zeigen demgegenüber eine etwas andere Entwicklung: Der Hauptrückgang des Laubmischbestandes fand erst von 1870 bis 1930 statt. Für diese merkwürdige Pha- 


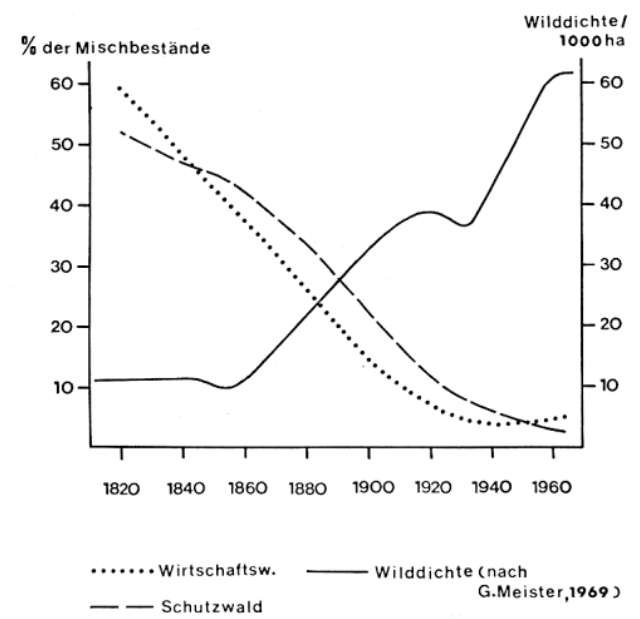

Abb.3: Anteil der Mischbestände in den Wirtschafts- und Schutzwäldern des Staatswaldes im bayerischen Hochgebirge und die Entwicklung der Wilddichte (nach G. MEISTER, 1969).

Proportion of mixed-stands in the commercial and protective forests within the stateowned forests in the $\mathrm{Ba}$ varian high mountainous area and the development of wildlife densities

senverzögerung in dem Rückgang der Laubhölzer läßt sich keine stichhaltige Erklärung finden, denn tatsächlich müßte die Degradation durch Waldweide und Äsung im Schutzwald und im Wirtschaftswald parallel verlaufen. Die Verzögerung des Laubholzrückganges in den Wirtschaftswäldern seit 1900 und der Anstieg seit 1950 erklären sich aus der verstärkten Einbringung und Förderung von Laubhölzern. In den Schutzwäldern wirkten sich dagegen Waldweide und Wildäsung ungehindert aus.

Heute läßt sich die Waldsituation für das bayerische Hochgebirge im Allgemeinen und im speziellen für den Raum Garmisch-Partenkirchen wie folgt charakterisieren: gestörte Verjüngung und Verfichtung im Montanwald, fehlende Verjüngung, Überalterung und damit Vergreisung im subalpinen Gebirgswald. Die bereits geschilderten erheblichen Äsungs- und Waldweideschäden im Gebirgswald haben stellenweise die Strauchschicht völlig vernichtet und den Jungwuchs praktisch unmöglich gemacht; der Wald lichtet sich zusehends. An die Stelle der absterbenden alten Gebirgsfichten kann kein Jungwuchs mehr treten. Man spricht in diesem Zusammenhang von der , Vergreisung“ des Waldes.

Nach einer eingehenden Luftbildauswertung der oberbayerischen Berg- und Gebirgswälder, durchgeführt von der Bayerischen Landesstelle für Gewässerkunde in München, soll nahezu ein Drittel der Waldbestände von der schleichenden Entwaldung betroffen sein (J. KARL, 1968). Örtlich haben wir es bereits nicht mehr mit einem echten Wald $\mathrm{zu}$ tun, sondern nur mehr mit einer bewaldeten Weide, ähnlich der schweizerischen Wytweide oder der französischen pâturage boisé.

Studiert man die Altersklassenstruktur des Wirtschaftswaldes im Raum Garmisch-Partenkirchen, so fällt der überwiegende Anteil des Altholzes (über 100jährig) auf: $66 \%$ sind älter als 100 Jahre, 34\% des Baumbestandes sind sogar älter als 140 Jahre (s. Tab. 2).

Bei den Nichtwirtschaftswäldern dürfte das Altholz noch stärker vertreten sein. Daraus ergibt sich zwangsläufig, daß in den nächsten 20-30 Jahren rund 40\% des heutigen Waldes zusammenbrechen werden. Damit dürfte es zu einer erneuten Absenkung der Waldgrenze auf etwa 1.200-1.400 m mit allen landschaftshaushaltlichen Folgen kommen (dazu W. DANZ, J. KARL u. H. TOLDRIAN, 1971). Gleichzeitig wird sich der montane Bergwald örtlich stark auflösen. Die gesamte Waldstufe um Garmisch-Partenkirchen, welche letztlich in ihrer Gesamtheit Schutzfunktion ausübt, wird die Sicherung des Talraumes nicht mehr gewährleisten können. Der einst zu diesem Zweck gesondert ausgeschiedene Schutzwaldgürtel, der mit nur 9\% der gesamten Waldfläche keinesfalls einen geschlossenen Gürtel darstellt, kann bereits seit langem seine Schutzfunktion nicht mehr erfüllen.

Neben der Vergreisung der Berg- und Gebirgswälder spielt die Verfichtung im Untersuchungsgebiet eine wichtige Rolle. Unter Verfichtung versteht man bekanntlich die ausgesprochene und unnatürliche Vorherrschaft der Fichte im Waldbestand. Im Raum Garmisch-Partenkirchen ist die seit 1810 zunehmende Verfichtung (Abb. 4) nicht wie im Alpenvorland auf öko-

Tabelle 2

\begin{tabular}{|c|c|c|c|c|c|c|c|c|}
\hline Altersklassen & I & II & III & IV & V & VI & VII & VIII \\
\hline Jahre & -20 & $21-40$ & $41-60$ & $61-80$ & $81-100$ & $101-120$ & $121-140$ & +140 \\
\hline $\begin{array}{l}\text { FA Garmisch-P. } \\
(1976)\end{array}$ & 15,5 & 18,7 & 10,2 & 6,0 & 3,4 & 4,0 & 8,0 & 34,1 \\
\hline FA Partenk. 195 & & & & & & & & \\
\hline Ges. & 10,6 & 7,6 & 4,5 & 1,3 & 4,4 & 13,6 & 14,1 & 38,5 \\
\hline „: Schutzwald & 2,6 & 0,3 & 1,2 & 0,1 & 4,6 & 19,1 & 25,9 & 40,3 \\
\hline
\end{tabular}




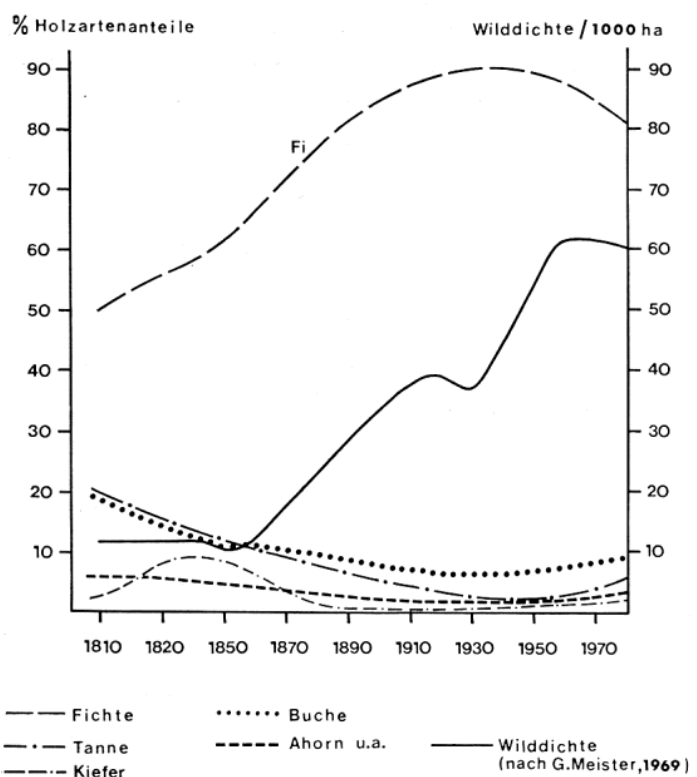

Abb.4: Entwicklungstendenz der Baumarten des FA Garmisch-Partenkirchen (in \% aller im jeweiligen Jahr entstandenen Bestände)

Development tendencies of trees within the boundaries of the Garmisch-Partenkirchen forest-unit (in \% of all stands developed in each year) nomische Gründe zurückzuführen. Sie ist ausschließlich durch die Waldweide und durch den hohen Wildbestand verursacht.

G. MEISTER, 1969 ermittelte im Vergleich mit Studien von R. MAGIN, 1949 und v. BüLOW, 1962 das ursprüngliche Baumartenverhältnis für das oberbayerische Gebirge. Für die Standortseinheit (Kalk) wird demnach ein Baumartenverhältnis von Fichte $45 \%$ zu Tanne $20 \%$ zu Buche $35 \%$ unterstellt, für die Standortseinheit II (Lias, Flysch) Fi 50 - Ta 40 - Bu 20. Tatsächlich beträgt jedoch das heutige Baumartenverhältnis im Untersuchungsgebiet Fi 73 - Ta 12 - Bu 15. Folgende Aufstellung (Tabelle 3) zeigt die einzelnen

\section{Tabelle 3}

\begin{tabular}{lccl}
\hline $\begin{array}{l}\text { Holzartenzusammensetzung } \\
\text { (Wirtschaftswälder) }\end{array}$ & Fichte & $\begin{array}{l}\text { Tanne } \\
\text { (in \%) }\end{array}$ & $\begin{array}{l}\text { Buche } \\
\text { u. a. }\end{array}$ \\
\hline $\begin{array}{l}\text { a Oberbayer. Gebirgsraum: } \\
\text { ursprünglich }^{1} \text { ) }\end{array}$ & 45 & 25 & 30 \\
heute $^{1}$ ) & 71 & 6 & 23 \\
montaner Bergwald $^{2}$ ) & 63 & 5 & 32 \\
subalp. Gebirgswald $^{2}$ ) & 76 & 3 & 21 \\
b Raum Garmisch-Partenk., $^{\text {gaum. }}$ & 73 & 12 & 15 \\
$\quad$ ges. & 85 & 7 & 8 \\
\hline subalp. Gebirgswald & & & \\
\hline
\end{tabular}

1) Nach MEISTER, 1969.

2) Nach DANZ, KARL u. TOLDRIAN, 1971.

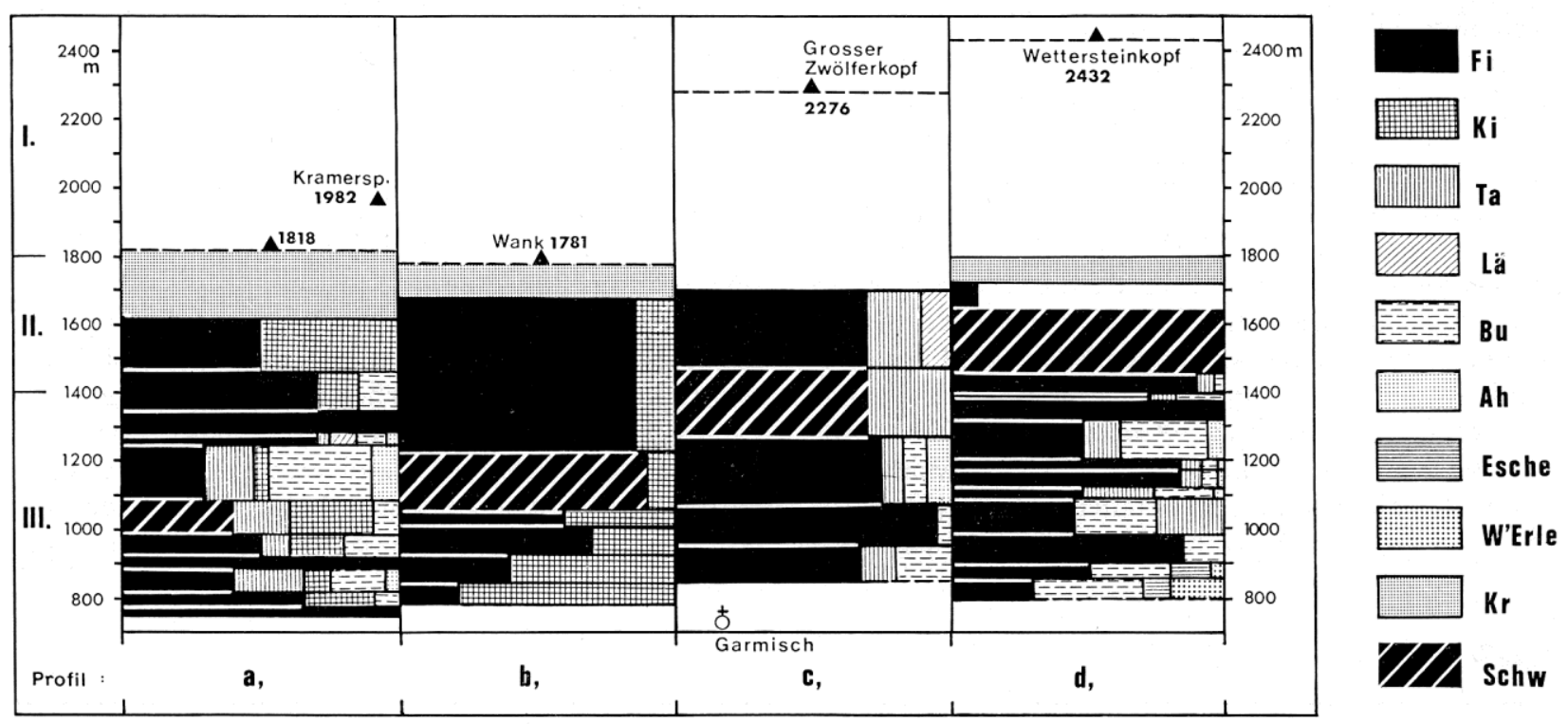

Abb. 5: Die Vertikalabfolge der Waldgesellschaften um Garmisch-Partenkirchen

Profile: a, Kramer S-Exposition (Loisach/Kögerlaine-Kramersteig; b, Wank-SW-Exposition (Wankseilbahn); c Waxenstein N-Exposition (Aigen-Gr. Zwölferkopf); d, Wetterstein N-Exposition (Partnach/Ferchenbach - Wettersteinkopf) Baumarten: $\mathrm{Fi}=$ Fichte, $\mathrm{Ki}=$ Kiefer, $\mathrm{Ta}=$ Tanne, Lä $=$ Lärche, $\mathrm{Bu}=$ Buche, $\mathrm{Ah}=$ Ahorn, W'Erle =Weißerle, $\mathrm{Kr}=$ Krummholz, Schw $=$ Schutzwald

$\mathrm{I}=$ alpine Stufe, $\mathrm{II}=$ subalpiner Gebirgswald, $\mathrm{III}=$ montaner Bergwald

In den einzelnen Waldgesellschaften der Profile sind die Hauptholzarten anteilmäßig (in \%) dargestellt Vertical composition of forest associations near Garmisch-Partenkirchen. Profiles of individual forest associations show percentage of principal timber species. 
Angaben der Holzartenzusammenstellung für die Wirtschaftswälder des oberbayerischen Hochgebirges und im Raum Garmisch-Partenkirchen.

Glücklicherweise ist die Verfichtung im Untersuchungsgebiet jedoch nicht überall gleich hoch, vielmehr wirkten die Expositionsunterschiede sehr stark auf die Wildäsung und damit auf den Waldaufbau. Die verschiedenen Zustandsstufen dieses vielgestaltigen Waldmosaiks (Abb. 5) kann man mit Hilfe der Waldzustandskarte 1: 25.000 (Bayer. Landesstelle für Gewässerkunde, München) und den Nutzungskarten 1: 10.000 des FA Garmisch-Partenkirchen gut ermitteln. Die Karte (Beil. II) „Ausmaß und Intensität der Waldgefährdung um Garmisch-Partenkirchen“ ermöglicht eine detaillierte Flächenübersicht der besonders verfichteten und ökologisch instabilen Räume innerhalb des Untersuchungsgebietes. Dabei wurde davon ausgegangen, daß bis zu 50\% Verfichtung eines Waldes als ökologisch vertretbar sind. Alle Waldflächen mit einem höheren Fichtenanteil (50-75\% und 75-100\%) wurden in die Karte aufgenommen. Da sich mit zunehmendem Fichtenanteil die ökologische Instabilität des Standorts durch stark verminderte Wasserspeicherkapazität erhöht, müssen die Waldgebiete, insbesondere bei aufgelockerter Bestockung, als ausgesprochen gestört und erosionsfördernd gelten. Beispielsweise sind am Kramerstock ca. 34\% der Waldfläche mehr als $50 \%$ verfichtet, am Wank sogar 40\%. Die im Untersuchungsgebiet überwiegend nordseitig exponierte Abdachung des Wettersteinmassivs zeigt dagegen nur
27\% Verfichtung. Insgesamt sind im Raum GarmischPartenkirchen im Mittel 31\% der Waldfläche sehr stark verfichtet. Der Fichtenanteil von $73 \%$ in den Wirtschaftswäldern ist demnach für das gesamte Untersuchungsgebiet nicht repräsentativ.

Für den Wald einer Gebirgslandschaft muß die Verfichtung von einem Drittel der Gesamtwaldfläche eine erhebliche Störung des hier ohnedies empfindlicheren Naturhaushaltes bedeuten. Um den Gesamtzustand der Landschaft zu erfassen, müssen noch weitere Parameter beachtet werden. $\mathrm{Zu}$ diesem Zweck wurde $\mathrm{zu}$ der Verfichtung die Bestockung in Bezug gesetzt (Abb.6) und drei Waldzustandsstufen der Gefährdung unterschieden: a) kritischer Waldzustand: Bestockung kleiner 0,5-0,5, b) sehr gefährdeter Waldzustand: Bestockung 0,6-1 bei Verfichtung von 50-100\% und c) höchstgefährdeter Waldzustand: Bestockung kleiner 0,3-0,5, Verfichtung 50-100\%. In Tabelle 4 sind die auf diese Weise ermittelten Werte des gefährdeten Waldanteils in Prozent des Gesamtwaldes aufgeführt und zwar nach Teillandschaft bzw. Waldstufe.

Die gesamte Waldfläche des Untersuchungsgebietes beträgt $102 \mathrm{~km}^{2}$ bei einem Bewaldungsprozeß von 55 . Von diesem Waldbestand sind $57 \%$ in einem kritischen bis höchst gefährdeten Zustand. Während von dem montanen Bergmischwald ( $84 \%$ d. ges. Waldes) nur knapp die Hälfte (49\%) einen unbefriedigenden Aufbau zeigt, hat sich fast der gesamte subalpine Gebirgswald (95\%) in seinem Aufbau und Zustand negativ verändert. Der Schutzwald mit nur 9\% der Ge-

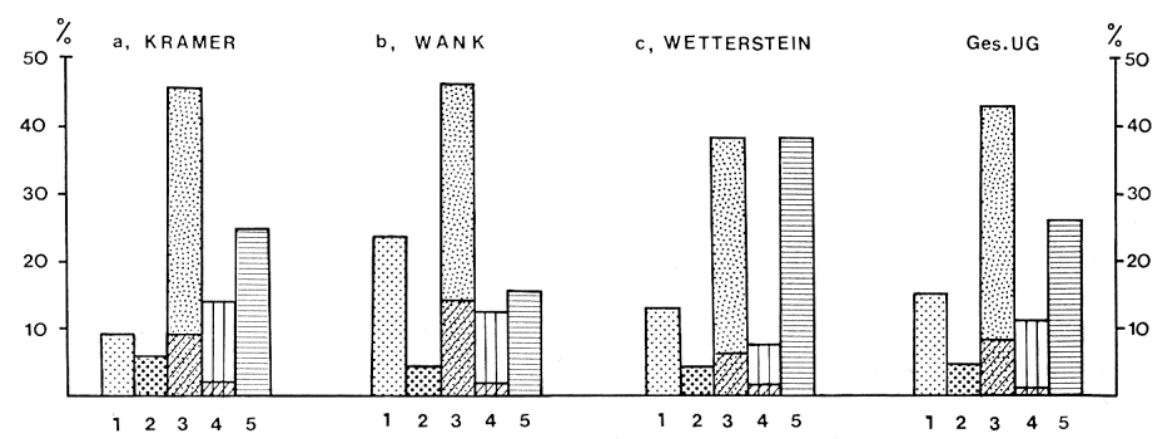

Abb.6: Der aktuelle Waldzustand im Raum Garmisch-Partenkirchen

1 waldfreies Kulturland; 2 ökolog. stabiler Bergwald; 3 ökolog. gestörter Bergwald; 4 subalpiner Gebirgswald; 5

Ödland; bei 3 und 4 Schutzwaldanteil schraffiert.

Ecological situation and arrangement of vertical forest zones in the Garmisch-Partenkirchen area

1 non-wooded, cultivated lands; 2 ecologically sound mountain forest of lower elevations; 3 ecologically disturbed

mountain forests of lower elevations; 4 subalpine high mountain forests; 5 wasteland; 3 and 4 proportion of protection forests shaded

Tabelle 4

\begin{tabular}{lcccl}
\hline & Kramer & Wank & Wetterstein & UG-gesamt \\
\hline Montan Bergwald & $54 \%$ & $69 \%$ & $42 \%$ & $49 \%\left(42,3 \mathrm{~km}^{2}\right)$ \\
Subalp. Gebirgswald & $100 \%$ & $90 \%$ & $89 \%$ & $95 \%\left(16 \mathrm{~km}^{2}\right)$ \\
\hline
\end{tabular}




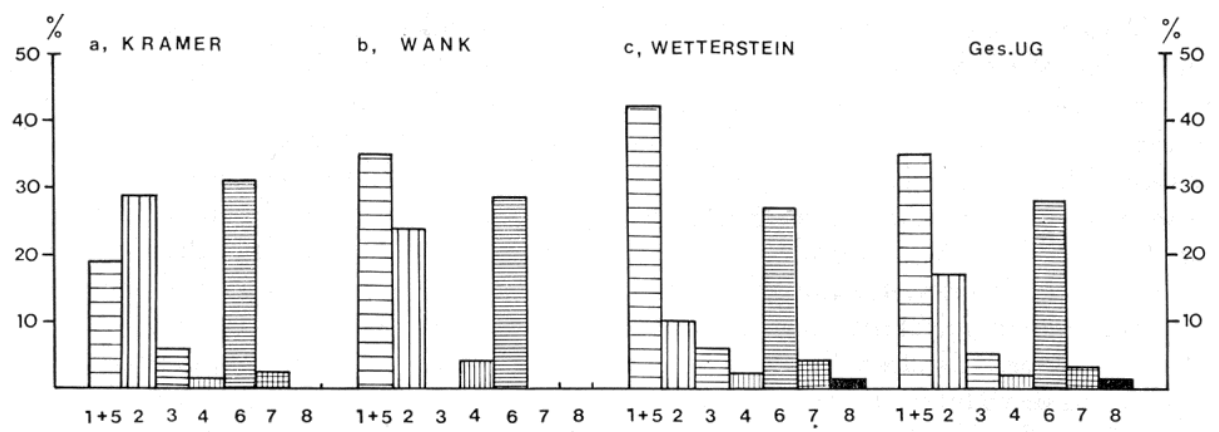

$A b b, 7:$ Aktuelle und potentielle Erosionsschäden im Raum Garmisch-Partenkirchen (nach Unterlagen v. J. KARL u. W. HöLTL, 1974)

\author{
$1+5$ geringe aktuelle $u$. potentielle Schäden; \\ aktuelle Schäden: 2 deutliche, 3 erhebliche, 4 starke; \\ potentielle Schäden: 6 deutliche, 7 erhebliche, 8 starke. \\ Current and potential erosion damage in the Garmisch-Partenkirchen area \\ $1+5$ little present and potential damages; \\ present damages: 2 obvious, 3 substantial, 4 extreme; \\ potential damages: 6 obvious, 7 substantial, 8 extreme
}

samtwaldfläche ist von diesem ungünstigen Zustand gleichermaßen betroffen.

Da fast der gesamte subalpine Gebirgswald starke Auflösungserscheinungen zeigt, ist der bereits erheblich gestörte montane Bergmischwald erhöht gefährdet. Ein wirksamer und nachhaltiger Schutz des Wirtschafts- und Kulturraumes von Garmisch-Partenkirchen durch den Waldgürtel ist daher nicht mehr gegeben. Durch die erneut zunehmende Schaf-Waldweide und die bislang nicht reduzierte hohe Walddichte wird sich die Gefährdung des Talraumes weiterhin erhöhen.

In diesem Zusammenhang interessiert ferner die Erosionsneigung der Landschaft. Ausgehend von der Tatsache, daß ein wesentliches Kriterium für die Zerstörung der Landschaft die Erosion ist, analysierten J. KARL u. W. HÖLTL, 1974 vermittels eines homogenen Rasterfeldes großräumig das oberbayerische Alpengebiet. Sie setzten die ökologischen Parameter Erosion, Vegetation, Gesteinsfestigkeit und Flußdichte zueinander in Relation und ermittelten auf diese Weise die aktuellen und die potentiellen Erosionsschäden.

Aus den Computerkarten dieser Studie wurden für den Raum Garmisch-Partenkirchen die entsprechenden Daten entnommen. Sie vermitteln einen guten Überblick der gegenwärtigen Belastung bzw. der noch vorhandenen Stabilität dieser Gebirgslandschaft (Abb. 7). Demnach sind nur 36\% des Untersuchungsgebietes als relativ stabil anzusehen, während $56 \%$ ökologisch gestört sind. Von diesen 56\% zeigen rund 1/4 (24\%) Erosionserscheinungen in Gestalt von Blaiken und Uferanbrüchen, Hangrutschungen etc. Weitere $32 \%$ sind landschaftsökologisch nicht mehr stabil und somit erosionsgefährdet. Unsere oben gemachte Aussage, wonach über $57 \%$ der Waldfläche bzw. $31 \%$ des Gesamtraumes sich in einem kritischen bzw. höchstgefährdeten Zustand befinden, bestätigt sich hiermit.

\section{Objektschutz und Landschaftssanierung}

Bereits 1788 erkannte der Osterreicher GRAF V. SAUER den direkten Zusammenhang zwischen zunehmender Bergbevölkerung, Vegetations- bzw. Walddegradation infolge verstärkter Waldweide und erhöhter Erosionsneigung und Wildbachtätigkeit. Zur gleichen Zeit setzte in Frankreich mit FABRE 1797 und LECREULS, 1804, in Osterreich mit G. v. ARETIN, 1808 und J. DuILE, 1826 die Erforschung des Wildbachphänomens ein.

Unter einem Wildbach (franz. torrent, ital. torrente) wird ein Gebirgsbach verstanden, welcher eine unausgeglichene Wasserführung mit extremen Abflußspitzen, ein sehr starkes Gefälle mit verstärkter Tiefen- und Seitenerosion und eine erhebliche Geschiebeführung aufweist. Wildbäche finden sich seit dem Bevölkerungsmaximum im 19. Jh. im gesamten Alpenraum und dementsprechend kann man eine Vielzahl von Wildbachtypen unterscheiden. Erstmals versuchten J. KARL u. J. MANGELSDORF, 1975 eine einheitliche Wildbachtypisierung der Ostalpen. Neben Wildbächen, deren Abfluß und Abtrag anthropogen nicht beeinflußbar sind, unterscheiden sie Wildbäche, die teilweise oder ganz beeinflußbar sind. Sämtliche Gebirgsbäche des Raumes Garmisch-Partenkirchen gehören zur Gruppe der beeinflußbaren Wildbäche. Sie sind letztlich eine Folge der Walddegradation infolge der Waldweide und des überhöhten Wildbestandes.

In der Karte „Belastung der Landschaft und Teilsanierung um Garmisch-Partenkirchen" sind sämtliche Wildbäche mit ihren Verbauungen nach den Unterlagen des Wasserwirtschaftsamtes Weilheim/Obb. kartiert. Erstaunlicherweise sind jedoch einige dieser verbauten Wildbäche in der amtlichen „Karte der Wildbäche, 1:25.000" (Bayer. Landesstelle f. Gewässer- 


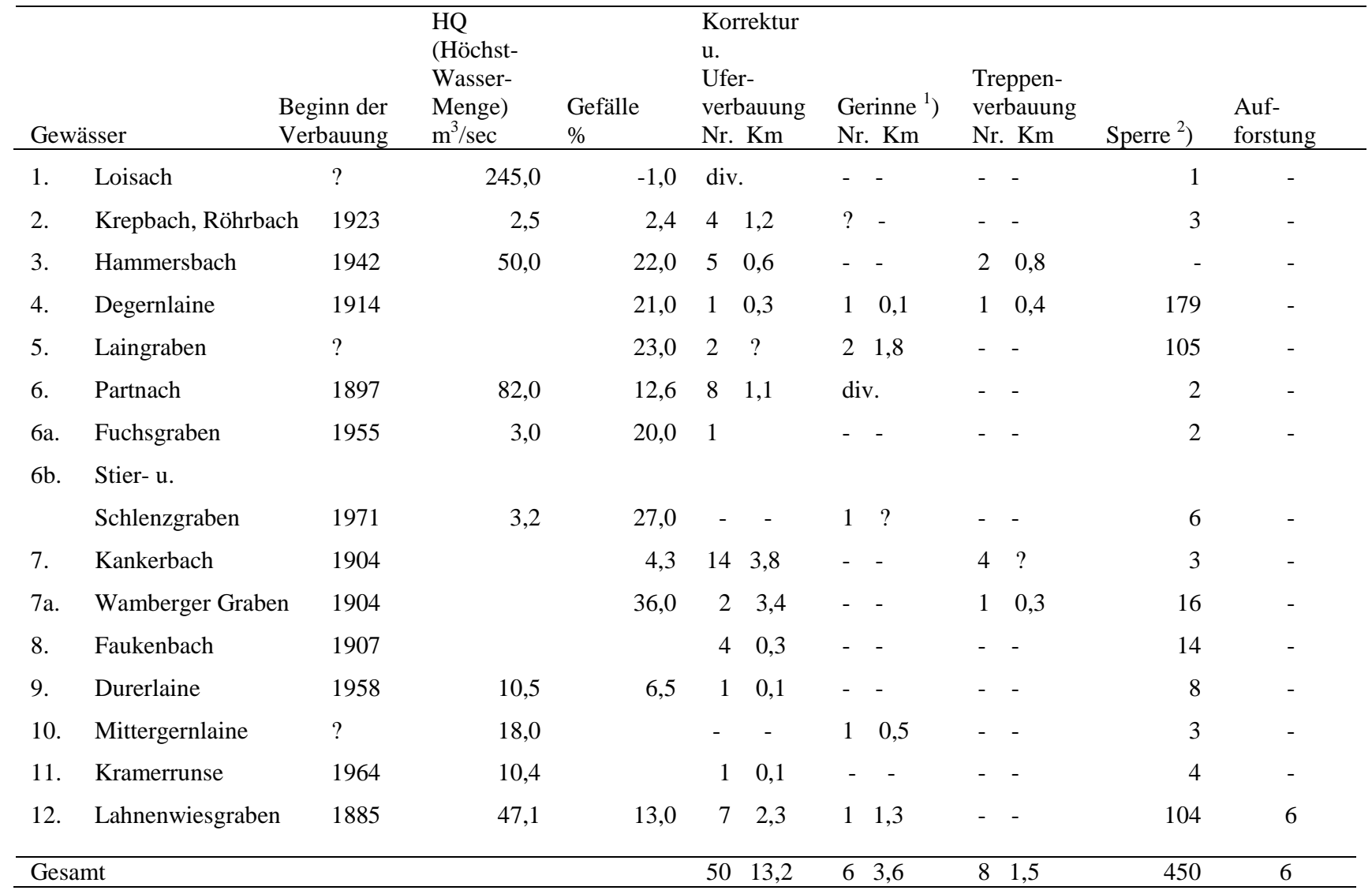

$\left.{ }^{1}\right)$ Gemauerte oder gepflasterte Gerinne.

$\left.{ }^{2}\right)$ Stein- und Holzsperren; Schwellen nicht erfaßt.

kunde, München, BI. 8532-8632 u. BI. 8432) nicht als Wildbäche gekennzeichnet, wie z. B. Rohr- und Krepbach, Hammersbach, Degernlaine, Laingraben, Kankerbach und Faukenbach.

Die ersten Wildbachverbauungen begannen im Raum Garmisch-Partenkirchen um 1890. Die Holzwerke jener frühen Bauphase sind jedoch nicht mehr zu lokalisieren, da sie entweder überschottert oder aber zerfallen sind. Seitdem wurden ca. 514 Baumaßnahmen zur Wildbachverbauung durchgeführt.

In der Tabelle 5 sind die verschiedenen Erneuerungsund Ausbesserungsarbeiten nicht aufgeführt, die z. T. erhebliche Kosten verursachten. Beispielsweise entstanden allein im Lahnenwiesgraben nach dem Hochwasser von 1959 Ausbesserungskosten in Höhe von 60.0000 DM. Seit Beginn der Wildbachverbauung im Untersuchungsgebiet dürften schätzungsweise weit über 100 Mio. DM für diese Verbauungen aufgewendet worden sein. Gegenwärtig entfallen auf den $\mathrm{km}^{2}$ des $\mathrm{zu}$ schützenden Talraumes 30 Baumaßnahmen bzw. 26 Sperren, umgerechnet zahlreicher weiterer Schutzmaßnahmen. Dennoch konnte trotz dieses beachtlichen technischen und finanziellen Aufwandes die Wildbachgefährdung nicht unterbunden werden. Selbst für die völlig verbauten Wildbäche wie Degernlaine und Laingraben kann die Gefahr eines erneuten Ausbrechens bzw. einer Vermurung nicht ausgeschlossen werden. Die Ursache für diese Situation liegt letztlich darin, daß man bis in die jüngste Vergangenheit reinen Objektschutz betrieb. In Frankreich dagegen, wo man nach den Erkenntnissen GRAF v. SAUER's bzw. jenen von A. SURELL 1841 nicht nur eine Wildbachverbauung, sondern eine umfassende Landschaftssanierung durchführte, beobachtete man sehr bald nach der Ausführung der Sanierungsarbeiten einen Rückgang der Wildbachtätigkeit.

Mit dem Bayerischen Wassergesetz vom 26.7.1962 wurde von der bislang ausgeübten Praxis des Objektschutzes durch die Wildbachverbauung zu der sog. Integralsanierung bzw. ganzheitlichen Sanierung übergegangen. Als Raumordnungsmaßnahme hat die Integralsanierung die Wiederherstellung des SollZustandes eines Landschaftsraumes durchzuführen. Darunter ist in erster Linie die Ausgleichung der Abflußverhältnisse und die Stabilisierung der Bodenerosion zu verstehen. Die einzelnen Maßnahmen der Integralsanie- 
rung (Österreich: Integralmelioration, Frankreich: travaux de restauration du terrain en montagne) sind folgende ( $\mathrm{J}$. KARL, 1972; H. MAYER, 1972):

- Anhebung der Waldgrenze durch Hochlagenaufforstung

- Umbau der Fichtenreinbestände in artenreiche

Mischbestände und Wiederbegrünung durch Aufforstung

- Entwässerung vernäßter und rutschgefährdeter

Hanglagen

- Trennung von Wald und Weide

- Punktuelle Baumaßnahmen mit sofortiger

Schutzwirkung wie Konsolidierung der Wildbäche durch Hart- und Lebendverbauung u. a.

Seit 1962 konnte im oberbayerischen Alpenraum aus Finanzgründen jedoch nur nach Prioritäten saniert werden, wie beispielsweise im Halblechgebiet. In anderen Gebieten, darunter auch der Raum GarmischPartenkirchen, ging man davon aus, daß die Gesamtsituation weniger kritisch ist und ein reiner Objektschutz ausreicht.

Nach dem ,Schutz dem Bergland - 10 Jahresprogramm Wildbachverbauung, 1973“" stehen von 1973 bis 1982 Finanzmittel lediglich für die dringendsten Maßnahmen der Wildbachverbauung und der Sanierung von Wildbacheinzugsgebieten bereit. Für das Untersuchungsgebiet wird der notwendige Planungsbedarf der Gesamtbaukosten auf 4,85 Mio. DM geschätzt, zusätzlich einer Sicherheitsrücklage von 1,35 Mio. DM. Auf technische Verbauungen entfallen davon 52\%, biologische Maßnahmen umfassen $16 \%$ und der Wegebau $32 \%$. Im Einzelnen beschränken sich die geplanten Maßnahmen auf drei Schwerpunkte: Laingraben, Faukenbach und Lahnwiesgraben.

Das bayerische Integralsanierungsprogramm ist jedoch nicht vollständig! Wie das Beispiel der Integralsanierung im Halblechgebiet zeigt, hängt dort der Erfolg der Maßnahmen (12,5 Mio. DM Investitionen) letztlich von der Lösung der Wildfrage ab. Bei der Lösung dieses Problems beschränkt man sich stattdessen weiterhin aus verschiedenen Gründen auf Ausweglösungen, wie die im Gebirge großflächig nicht praktikable Zäunung oder die Erweiterung der Äsungskapazität durch Schaffung von Wildäsungsflächen (Wildäcker und Äsungsgehölze). Die einzige Möglichkeit einer Lösung der Wildfrage besteht in der Reduzierung des Schalenwildes durch den Abschuß, wie er in Österreich bei Winterfütterungen erfolgreich durchgeführt wird. Grundsätzlich muß in diesem Zusammenhang darauf hingewiesen werden, daß die Reduzierung des Schalenwildes auf das „,natürliche“ $\mathrm{Maß}$, wie sie heute von forstlicher Seite verschiedentlich gefordert wird, nicht der aktuellen ökologischen Situation des Waldes entspricht. Tatsächlich haben sich, wie gezeigt wurde, die Waldbiotype seit 1850 sehr stark verändert. Selbst die relativ niedere Wilddichte vor 1840, welche heute als natürlich gilt, dürfte noch zu hoch sein.
Der Einwand, daß der voraussichtliche Bevölkerungsrückgang in Deutschland eine Gesundung des degradierten Berg- und Gebirgswaldes und damit der Landschaft zur Folge hat und eine Integralsanierung demzufolge kaum mehr nötig sei, selbst wenn das Problem der Waldweide und der Wilddichte gelöst würde, ist leider für Bayern nicht zutreffend. Durch den innerdeutschen und ausländischen Wanderungsgewinn dürfte die Bevölkerung Bayerns bis zum Jahr 2030 sich tatsächlich um 10\% erhöhen (W. ISTEL, 1975). Mit der zu erwartenden weiteren Verstärkung des Fremdenverkehrs im Raum Garmisch-Partenkirchen dürfte sich auch die bislang nur punktuelle Landschaftsbelastung ausweiten. Somit käme zu der Waldbelastung durch Waldweide und Wildäsung noch der Fremdenverkehr, der allerdings kaum eine derartige Flächendegradation verursachen kann.

\section{Folgerungen}

Die am Beispiel des alpinen Landschaftsraumes Garmisch-Partenkirchen durchgeführten Untersuchungen ergaben, daß die Walddegradation und Erosionsgefährdung erheblich größer sind als bisher angenommen wurde. Durch Verfichtung und Vergreisung sind $49 \%$ des montanen Bergmischwaldes und 95\% des subalpinen Fichtengebirgswaldes ökologisch gestört und in ihrem Bestand z. T. hoch gefährdet. Nur etwa ein Drittel des Untersuchungsgebietes kann noch als ökologisch intakt gelten. Auf $24 \%$ der Gesamtfläche ist der Wald bereits so stark degradiert, daß Bodenerosion auftritt; weitere $32 \%$ zeigen potentielle Erosionsschäden. Als Hauptursache dieser Walddegradation wurden die Nachwirkungen bzw. eine neue Form der Waldweide und ein seit 1850 mindestens verzehnfachter und überhöhter Schalenwildbestand erkannt. Unter den gegebenen Umständen ist die Schutzfunktion des Waldgürtels nicht mehr gewährleistet, sie verringert sich vielmehr ständig in Richtung auf eine kritische Schwelle in etwa 20 bis 30 Jahren.

Anstatt des Objektschutzes in Gestalt der Wildbachverbauung muß die Integralsanierung sofort einsetzen, da ein Stabilisierungsprozeß erst mit einer Verzögerung von 30-40 Jahren voll wirksam wird. Die bereits begonnene Agrarleit- und Waldfunktionsplanung muß gegenüber der Integralsanierung zurückgestellt werden. Außerdem darf im Raum GarmischPartenkirchen die Integralsanierung nicht punktuell, sondern muß flächig durchgeführt werden, und zwar nach dem Nachhaltigkeitprinzip der Forstwirtschaft. Die Sanierungsarbeiten können jedoch nur dann auf Dauer erfolgreich sein, wenn die Regulierung der Schalenwilddichte als Kardinalpunkt in das bestehende Programm aufgenommen wird. Zu diesem Zweck müßte eine Erfassung und Kartierung der Äsungskapazität dieses Raumes und eine Methode zur exakten Bestimmung der Wilddichte entwickelt werden. 


\section{Literatur}

AUlitZKY, H.: Schutzfunktionen des Waldes in der Raumordnung. Ber. z. Raumforschung u. Raumplanung 13, 4, 1969, S. 9 ff.

- : Vorläufige Wildbach-Gefährlichkeitsklassifikation für Schwemmkegel. Österr. Wasserwirtschaft, Wien 1972a.

- : Die Entwicklung des forsttechnischen Systems der Wildbachverbauung. Österr. Wasserwirtschaft, Wien 1972b, S 183-192.

BAASEN, C.: Die Veränderung von Boden und Vegetation nach gcnerationsweisem Fichtenanbau. Diss. Hann.-Münden 1940.

Bayer. Staatsminist. d. Innern, Oberste Baubehörde (Hrsg.): Schutz dem Bergland. München 1973.

Bayer. Staatsminist. f. Ernährung, Landwirtsch. u. Forsten (Hrsg.): Wald und Forstwirtschaft in Bayern. München 1963. BuBENIK, A.: Grundlagen der Wildernährung. Berlin 1959.

- : Wilddichte - Fassungsvermögen - Wildschaden. Österr. Arbeitskreis für Wildtierforschung, Jb. 4, 1962/63.

BüLow, G. v.: Die Sudwälder von Reichenhall. Mitt. aus d. Staatsforstverwaltung Bayerns, München 1962.

DANZ, W.: Aspekte einer Raumordnung in den Alpen. WGIBerichte zur Regionalforschung, Bd. 1, München 1970.

- : Regelkreis alpine Umwelt: Konsequenz Integralplanung. In: Die Zukunft der Alpenregion, Hrsg.: H. Wichmann, München 1972, S. 166-171.

DANZ, W., J. KARL u. H. TOLDRIAN: über den Waldzustand im oberbayerischen Hochgebirge. Forstwiss. Centralblatt, Bd. 90, 1971, S. 87-103.

Demontzey, P.: Etude sur les travaux de reboisement et de gazonnement de montagne. Paris 1878, (Übersetzung: A. v. SECKENDORFF: Studien über die Arbeiten der Wiederbewaldung und Begrasung der Gebirge. Wien 1880).

DoposcheG, J.: Berge und Pflanzen in der Landschaft Werdenfels. Garmisch 1927.

Esser, W.: Beitrag zur Untersuchung der Äsung des Rehwildes Zschr. f. Jagdwiss. Bd. 4, 1958, S. 1-40.

FISCHBACHER, G.: Über das Waldweideproblem und die Bergbauernfrage. Bayer. Landwirtschaftl. Jahrbuch 1956, S. 199.

FRÖHLICH, J.: Wald und Wild im Urzustand, Allg, Forstztg. Bd. 66, Wien 1955, S. 192-193.

Fromme, G.: Der Waldrückgang im Oberinntal. Mitt. forstl. Bundesversuchsanstalt Mariabrunn, Bd. 54, Wien 1957.

GAMs, H.: Aus der Geschichte der Alpenwälder. DAV, Bd. 68,1937, S. 157-171.

GLÄSSER, E.: Zur Problematik der natürlichen und anthropogenen Vegetationsgesellschaften. Kurzbericht in: Umschau in Wissenschaft u. Technik v. 22. 10. 1970.

GRÖTZBACH, E.: Die Entwicklung der bayerischen Fremdenverkehrsgebiete in den letzten vierzig Jahren. Mitt. Geogr. Ges. München, Bd. 53, 1968, S. 267-292.

Helm, A. G.: Das Forstamt Partenkirchen. Diss. Freiburg i. Br.1952
HeRmes, K.: Die Lage der oberen Waldgrenze in den Gebirgen der Erde und ihr Abstand zur Schneegrenze. Kölner Geogr. Arb., H. 5,1955.

HiBLER, I.: Die bayerische Almwirtschaftsfrage mit besonderer Beziehung auf die Alm- und Weiderechtsgebiete. Garmisch 1910.

IsTEL, W.: Zur Frage einer zukünftigen strukturräumlichen Entwicklung bei stagnierender oder schrumpfender Bevölkerung. Raumforschung u. Raumordnung, Bd. 33, 1975, S. 71-80.

Jugoviz, R. A.: Wald und Weide in den Alpen. Wien 1908.

KARGL, J.: Die Almwirtschaft im Bezirk Garmisch. Maßnahmen zu ihrer Förderung. Diss. München 1946.

KARL, J.: Berglandschaft in Gefahr. Auf der Alpe, H. 20, S. 86ff. u. H. 21, S. 1ff., 1969.

- : Naturschutz und Wasserwirtschaft. In: Die Zukunft der Alpenregion, Hrsg. H. Wichmann, München 1972, S. 73 bis 92.

- : Sanierung Halblechgebiet als Integralmaßnahme im

Alpenraum. Garten und Landschaft, H. 1, München 1972, S. 1820.

Karl, J. u. W. DanZ: Der Einfluß des Menschen auf die Erosion im Bergland. Schr. Reihe Bayer. Landesst. Gewässerkunde, Bd. 1, München 1969.

KARL, J. u. W. HöLtL: Analyse alpiner Landschaft in einem homogenen Rasterfeld. Schr. Reihe Bayer. Landesst. Gewässerkunde, Bd. 10, München 1974.

KARL, J .u. J. MANGELSDORF: Die Wildbachtypen der Ostalpen. Interpraevent, Bd. 1 ,Wien 1975, S. 397-406.

KoBell v.: Wildanger. München 1958.

KRINER-FISCHER, E.: Garmisch-Partenkirchen, einst und jetzt. Diss. München 1930.

Kuoch, R. u. R. Amiet: Die Verjüngung im Bereich der oberen Waldgrenze der Alpen. Mitt. d. Schweiz. Anst. f. d. forstl. Versuchswesen, Bd. 46, H. 4, 1970, S. 165-328.

LAATSCH, W.: Bodenschutz im Bergwald des bayerischen Alpengebietes. Forstwiss. Centralbl., Bd. 90, 1971, S. 159 bis 174.

LAATSCH, W. u. W. GROTTENTHALER: Labilität und Sanierung der Hänge in der Alpenregion des Landkreises Miesbach. Bayer. Staatsministerium f. Ern., Landwirtsch. u. Forsten, München 1973.

MAGIN, R.: Der Einfluß der Waldweide im oberbayerischen Hochgebirge auf Boden, Zuwachs und Ertrag des Waldes. Diss. München 1949.

MANTEL, W.: Die Weideverhältnisse am Teisenberg und Sulzberg. Alm und Weide 1925.

MAYER, H.: Zur Waldgeschichte des Steinernen Meeres. Jb. d. Ver. zum Schutze d. Alpenpflanzen u. -tiere, 1965.

- : Möglichkeiten und Grenzen der Schalenwildhege im Gebirgswald. Schweiz. Ztschr. f. Forstw., Allg.

Forstztschr.1972.

- : Die Wälder des Ostalpenraumes. Stuttgart 1974.

MAYer, H. u. A. Steinhauser: Äsungswahl beim Gamswild. Allg. Forstztg., Bd. 78, Wien 1967, S. 21-26.

MeIster, G.: Ziele und Ergebnisse forstlicher Planung im oberbayerischen Hochgebirge. Forstwiss. Centralbl., Bd. 88, H. 2 u. H. 4,1969, S. $97 \mathrm{ff}$. 
- : Wald und Wild. Konflikt: Forstwirt-Jäger. In: Die Zukunft der Alpenregion, Hrsg.: H. Wichmann, München 1972, S. 116123.

- : Naturschutz und Jagd im Hochgebirge. In: Deutscher Alpenverein - Lehrschriftenreihe: "spleen, show, chance: Umweltschutz", München 1972, S. 93-118.

MElChIAR, J.: Das Abäsen der Waldvegetation durch das Rotund das Rehwild. Zschr. f. Jagdwiss., Bd. 6, 1960, S. $78 \mathrm{ff}$.

OBERDORFER, E.: Die Schafweide im Hochgebirge. Forstwiss. Centralbl., Bd. 70,1951, S. 117ff.

Pechmann, H. v.: Beiträge zur Geschichte der Forstwirtschaft im oberbayerischen Hochgebirge. Forstwiss. Centralbl., Bd. 18, 1932.

Röck, E.: Das Werdenfelser Land in früherer Zeit. Partenkirchen 1934.

RUBNER, K.: Die pflanzengeographischen Grundlagen des Waldbaus. Berlin 1953.

RuPPERT, K., L. Deuring u. J. MAIER: Das Bergbauerngebiet der deutschen Alpen. WGI-Berichte zur Regionalforschung, Bd. 7, München 1971.
SPEER, W.: Wild und Jagd im Zeitalter der Technik. Der Deutsche Jäger, H. 87, 1969.

STAUDER, S.: Methoden und Erfahrungen der Integralmelioration in Tirol. Allg. Forstztg., Bd. 79, H. 8, Wien 1968, S. 196-198.

STRELE, G.: Grundriß der Wildbach- und Lawinenverbauung. Wien 1950.

Surell, A.: Etude sur les torrents des Hautes-Alpes. Paris 1841. WATSON, D. W.: Forest or bog: man the deciding factor. In: The Scottish Geograph. Magazine, 1939, S. 148-161.

WEBER, A.: 70 Jahre forsttechnisches System der Wildbach- und Lawinenverbauung in Österreich. Allg. Forstztg., Bd. 65, Wien 1954, S. $281 \mathrm{ff}$.

WIESER, R. F.: Probleme der Walderhaltung im Hochgebirge. Allg. Forstztg., Bd. 68, Wien 1957.

ZöTTL, H.: Die Vegetationsentwicklung auf Felsschutt in der alpinen und subalpinen Stufe des Wettersteingebirges. Diss. München 1950.

\section{Anhang}

Beilage I: Ausmaß und Intensität der Waldgefährdung um Garmisch-Partenkirchen

Extent and intensity of forest-endangerment around Garmisch-Partenkirchen.

Beilage II: Belastung der Landschaft und Teilsanierung um Garmisch-Partenkirchen

Stress on the landscape and partial restoration around Garmisch-Partenkirchen. 
Ausmaß und Intensität der Waldgefährdung um Garmisch - Partenkirchen Extent and intensity of forest-endangerment around Garmisch-Partenkirchen
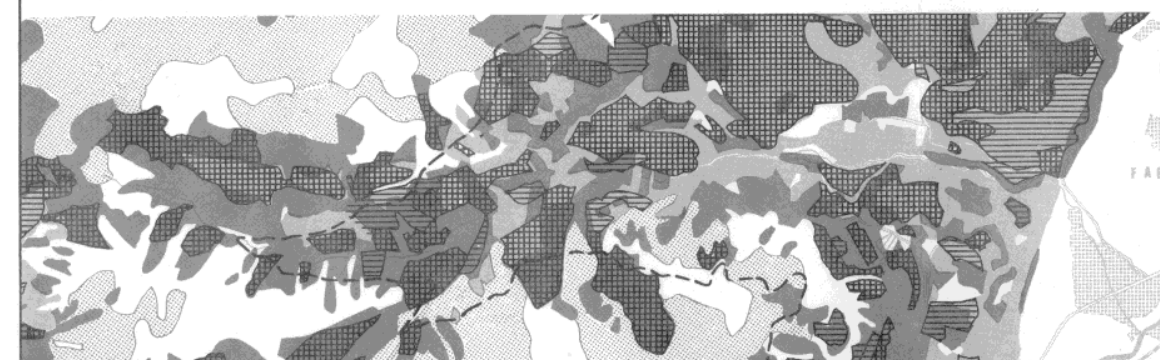

S

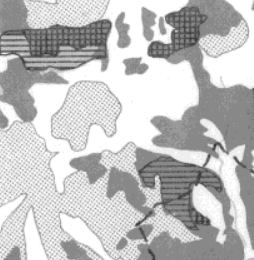

$1+\frac{1}{3}$

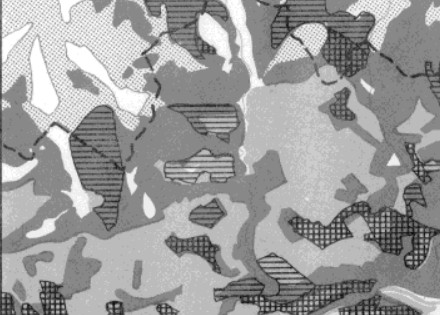

-
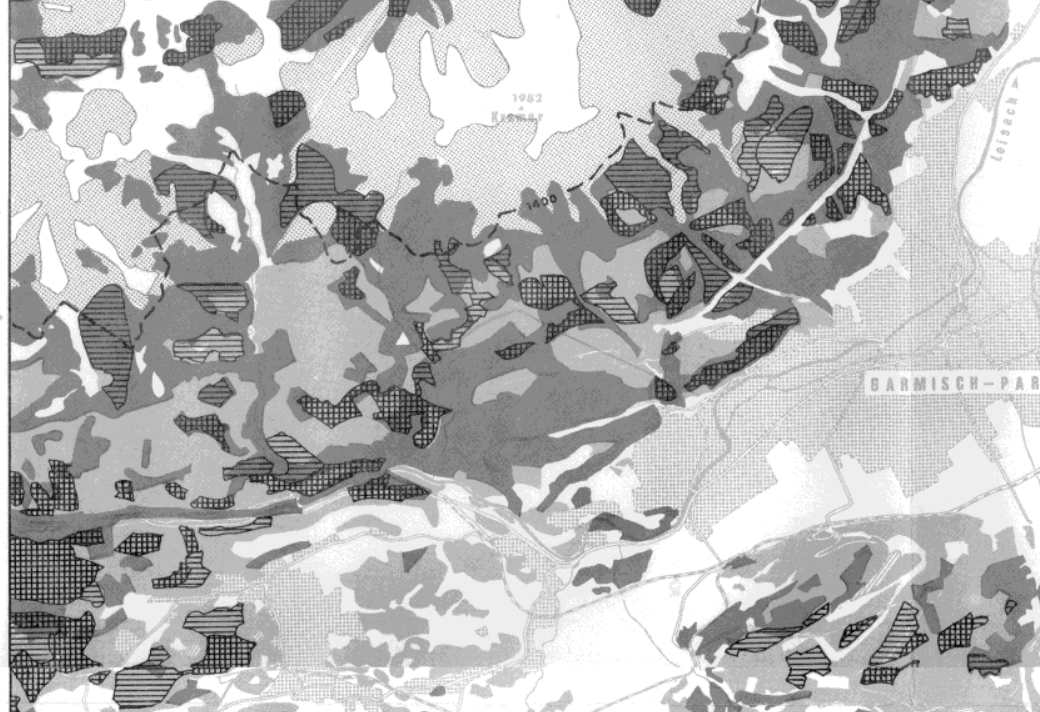

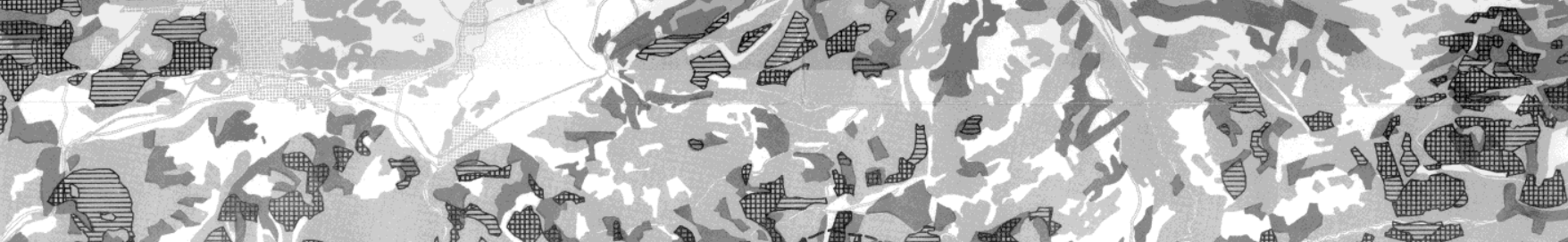

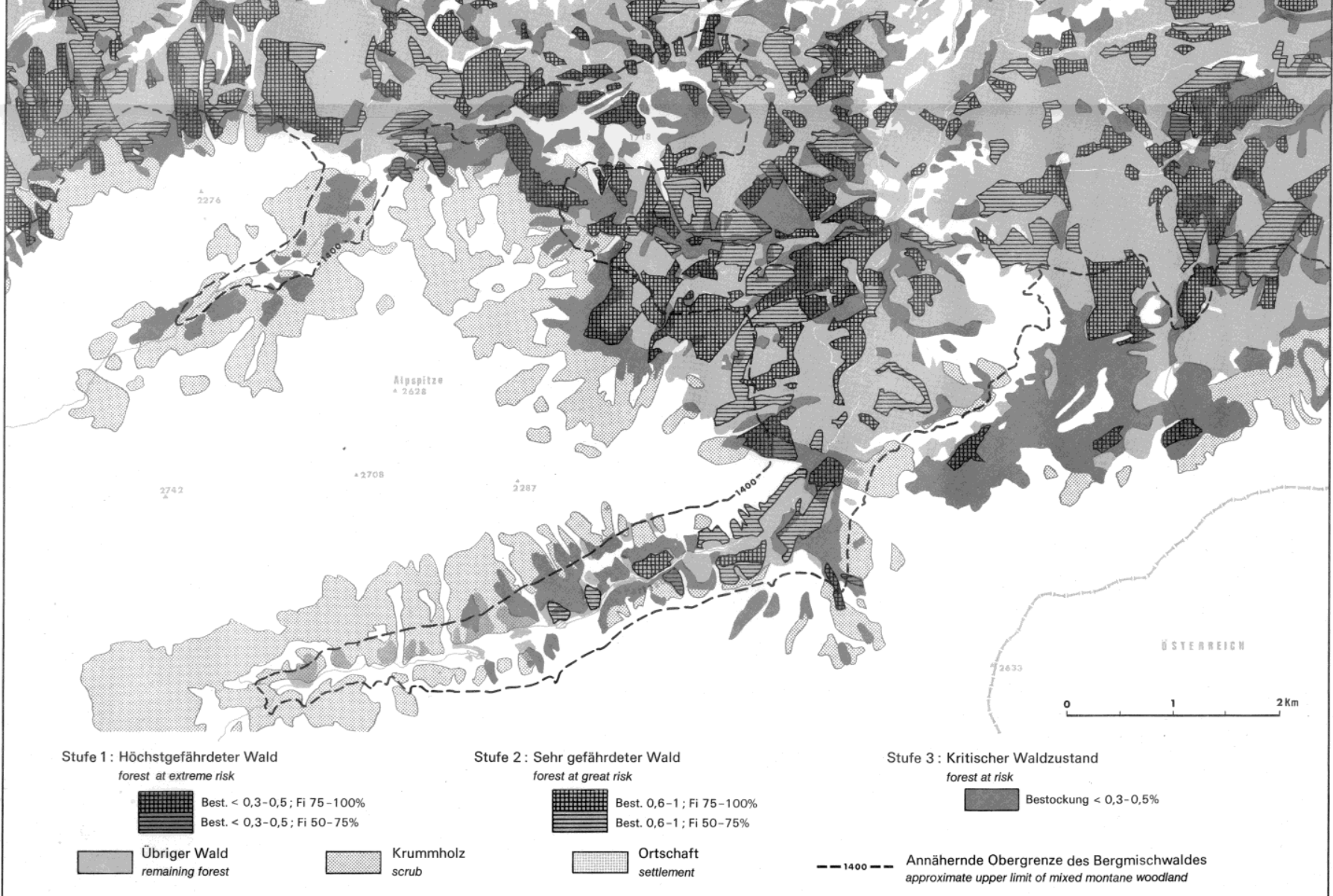

Quelle: Toldrian, H.: Karte des Waldzustandes. München 1972; Unterlagen des Forstamtes Garmisch-Partenkirchen 


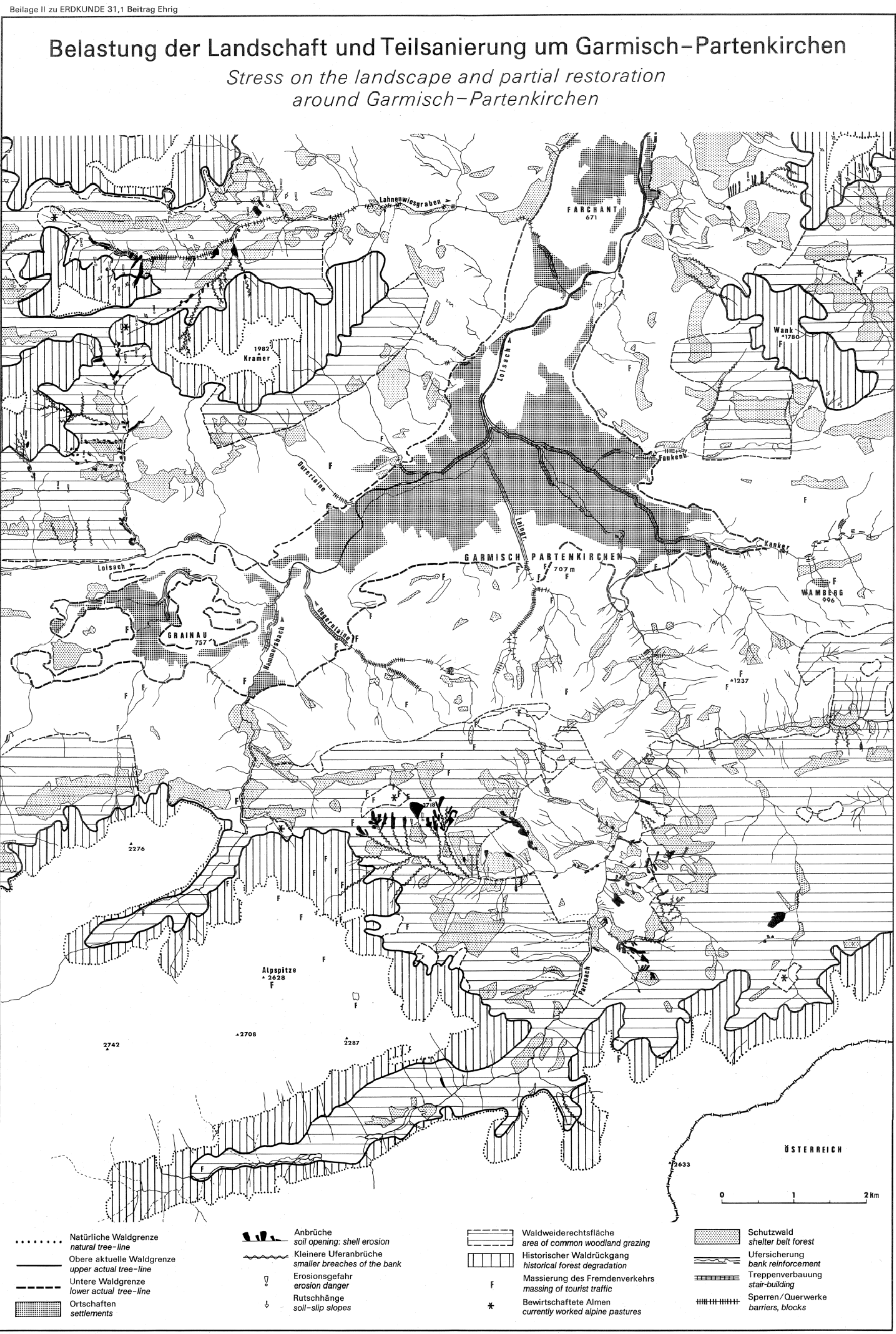

